\title{
How to write a scientific paper?
}

\author{
Mohey El-Din Ragab Elbanna, MD \\ Department of General Surgery, Ain-Shams University, Cairo, Egypt.
}

\section{Introduction:}

Scientific publishing is an essential prerequisite for a successful academic career. Scientific writing and publishing are essential for the progress of science. They are the formal way to convey scientific knowledge. Retesting the published knowledge, proving its value and credibility leads to building upon that knowledge. That is how the scientific pyramid is established and raised, block over block.

Beside these scientific and idealistic considerations, promotion to a higher university post, in our country, is based on evaluation of the published papers and the papers accepted for publication. However, writing a scientific paper is a skill that we learn on our own. There are no structured courses to teach scientific writing or paper writing. Luckily, I had a chance to attend the first course to be held in Egypt on manuscript writing, thanks to Prof. Dr. Alaa Ismail, professor of general surgery, Ain Shams University.

This review article aims to outline the bases of scientific paper writing. Herein, it is important to state that I believe it is only the beginning of an effort that has to continue and develop. We need to develop our research capabilities. The reasons for that are clear enough, and more than adequately discussed and talked about. The "How" question still waits for answers. This article is not discussing how to carry out surgical research. It is rather on how to write down and present the research results. It is a crucial skill that we need to master. I believe that we do a lot of good work. But we lack good documentation and good presentation. That is why we have to work on that basic professional requirement.

\section{The Need for Clarity:}

The key characteristic of scientific writing is clarity. Ideally, clarity should be a characteristic of all types of communications, yet in scientific writing clarity is an absolute demand. Scientific communication, as all types of communication, is a two-way process. Just as a signal of any kind is useless unless it is perceived, a published scientific paper is useless unless it is both received and understood by its intended audience.

Scientific writing is the transmission of a clear signal to a recipient. The words of the signal should as clear and simple and well ordered as possible. The flowery literary ornamentation is very likely to cause confusion and should be avoided in writing research papers. Many kinds of writing are designed for entertainment. Scientific writing has a different purpose: to communicate new scientific findings. Scientific writing should be as clear and simple as possible.

Consequently, we can consider a scientific experiment incomplete until the results have been published and understood.

\section{Definition of a scientific paper:}

A scientific paper is a written and published report describing original research results. That short definition must be qualified, however, by noting that a scientific paper must be written in a certain way and it must be published in a certain way. To properly define "scientific paper", we must define the mechanism that creates a scientific paper, i.e. primary publication. Abstracts, theses, conference reports, and many other types of literature are published, but such publications do not normally meet the criteria of primary publication.

The scientific primary paper must be the first disclosure. Certainly, first disclosure of new research data often takes place via oral presentation at a scientific meeting. However, effective first disclosure is accomplished only when the disclosure takes a form that allows the peers of the author, either now or in the future, to fully comprehend and use the disclosed data.

Thus, sufficient information must be presented so that potential users of the data can (1) assess observation, (2) repeat experiment, and (3) evaluate intellectual 
processes.

Certainly, electronic publishing meets the criteria of primary publication.

The form of a publication to be considered scientific and primary should be essentially permanent, made available to the scientific community without restriction, and made available to the information retrieval services (Biological abstracts, Index Medicus, etc.). Thus, publications like newsletters, corporate publications, and controlled-circulation journals, cannot be considered valid scientific publications for research papers, though many of them are of value for their readers.

On the other hand electronic publications that meet these criteria are considered primary publications.

\section{Organization of a scientific paper:}

A scientific paper is organized to meet the needs of valid publication. It is, or should be, highly stylized, with distinctive and clearly evident component parts. The most common labeling of the component parts is Introduction, Materials and Methods, Results, and Discussion, hence the acronym IMRAD. The IMRAD system was prescribed as a standard by the American National Standards Institute, first in 1972 and again in 1979.

There are journals that prefer other systems of organization. In such cases, organization of the paper is determined by reviewing the instructions to authors of that journal. The organization of the paper is the pillar of preparation of a scientific paper. It is the organization of the paper that distinguishes it from other literature.

The uniform organization of papers makes reading scientific papers an easier task. This is of special importance in an era when increasing numbers of papers are published, and the academic surgeon has to review a great number of them to keep up in his specialty.

\section{How to prepare the "Title"?}

In preparing the title of the paper, the author would do well to remember one fact: The title will be read by thousands of people. Perhaps, only a few people, if any, will read the entire paper, but many people will read the title, either in the original paper, or in one of the secondary publications (abstracts, indexing).
A good title is composed of the fewest possible words that adequately describe the contents of the paper. The title has to be accurate, concise and specific, summarizing the main point. It should not be too short or too long. This means it should be formed of the number of words required to define the issue raised and discussed in the paper. The author needs to avoid redundancy and overgeneralization.

Occasionally, titles are too short. In such cases, they do not tell much about the subject of study. Much more often, they are too long and long titles are less meaningful than short ones.

The title of the paper is a label. It is not a sentence. Because it is not a sentence, with the usual subject, verb, object arrangement, it is simpler than a sentence, and shorter. However, the order of the words is as important.

The meaning and order of the words in the title are of importance to the potential reader who sees the title in the journal table of contents. These considerations are equally important to all potential users of the literature, including those who become aware of the paper via secondary sources, as Index Medicus, and others. That is why it is important to provide the right key words in the title. That is the terms in the title should be limited to those words that highlight the significant content of the paper in terms that are both understandable and retrievable.

I should mention that some experienced writers prepare the title and abstract after the paper is written, even though by placement these elements come first.

However, it is better to put on paper a provisional title and an outline of the research paper.

\section{How to prepare the "Abstract"?}

An abstract should be considered a miniversion of the paper. It should provide a brief summary of each of the main sections of the paper: Introduction, Materials and Methods, Results, and Discussion. Houghton (1975) stated that "An abstract can be defined as a summary of the information in the document". Many authors prefer to write the abstract after they have finished writing the paper proper.

A well-prepared abstract enables readers to 
identify the basic content of a document quickly and accurately, to determine its relevance to their interests, and thus to decide whether they need to read it in its entirety" (American National Standards Institute, 1979b).

The number of words in the abstract is usually decided by the journal. If not the average number of words of the abstract is 250 words.

The abstract is either written as one paragraph, or presented in a structured form, consisting of a few paragraphs. In either form the abstract should (1) state the principle objectives and scope of the investigation, (2) describe the methods employed, (3) summarize the results, (4) state the principle conclusions.

Most or the entire Abstract should be written in the past tense as it describes work already done. The abstract should never give information or conclusion that is not stated in the paper. References to the literature must not be cited in the Abstract. Abbreviations should not be used in the abstract, except if a long term is used several times in the abstract. Abbreviations should be reserved for the text. The language of the abstract should be familiar to the potential reader.

As the abstract will be published in several forms, electronic and print, and will be read independent from the paper, it should be sufficient and self-contained.

\section{How to write the "Introduction"?}

The introduction is the first section of the text proper. The purpose of the introduction should be to supply sufficient background information to allow the reader to understand and evaluate the results of the present study without needing to refer to previous publications on the topic. The introduction should also provide the rationale for the present study. It should clearly state the purpose of writing the paper. References to use in the introduction are chosen carefully to provide the most important background information.

Much of the introduction must be written in the present tense, because you will be referring primarily to the problem of the paper, and the established knowledge relating to it at the start of the work.

Suggested rules for a good introduction are as follows: (1) The Introduction should present first, with all possible clarity, the nature and scope of the problem investigated. (2) It should review the pertinent literature to the reader. (3)It should state the method of the investigation and, if necessary, the reasons for the choice of a particular method should be stated. (4) The aim of the study should be stated in one or two sentences at the end of the introduction section.

In a sense, a scientific paper is like other types of journalism. In the introduction you should have a hook to gain the reader's attention. Why did you choose this subject, and why is it important?

\section{How to write the "Materials and Methods"?}

In the materials and methods section, you must give the full details of the methodology of the research. This section should be written in the past tense. The main purpose of the Materials and Methods section is to describe the experimental design and then provide enough detail so that a competent specialist can repeat the experiments.

Most readers will skip this section as they know the general methods used and they probably have no interest in the experimental detail. However, careful writing of this section is important because for the paper to be scientific, the results must be reproducible. Therefore, the author must provide the methods for repetition by others.

When the paper is subjected to review, a good reviewer will read the Materials and Methods carefully. If there is serious doubt that the experiments could be repeated, it may be rejected.

For materials, the exact technical specifications and quantities and source or method of preparation are included. Avoid the use of trade names; use of generic or chemical names is usually preferred. This avoids advertising for the producing company. Besides, the trade name is usually known only in the country of origin, while the generic name is more likely to be known throughout the world. However, in case of presence of known differences between propriety products, it is essential to mention the trade name and the name of the manufacturer.

Experimental animals and microorganisms should be identified accurately. Sources should be listed and special characteristics described. 
If human subjects are used, the criteria for selection should be described, and an "informed consent" statement should be added to the manuscript if required by the journal.

For methods, the usual order of presentation is chronological. Obviously, however, related methods should be described together, and straight chronological order cannot always be followed.

The Materials and Methods section usually has subheadings. When possible, subheadings must match those in the results section. That is why it is better to write the Materials and Methods and results together to maintain internal consistency. The reader will be able to find out quickly the relationship between both.

Statistical analyses are often necessary, but you should feature and discuss the data not the statistics. Generally, a lengthy description of statistical method indicates that the writer has recently acquired this information and believes that the readers need similar enlightenment. Ordinary statistical methods should be used without comment; advanced or unusual methods may require a literature citation.

In describing the methods, you should give sufficient details so that a competent worker could repeat the experiment. If the method is new, all of the needed details should be provided. However, if the method is standard or previously published in a standard journal, only the literature reference should be given.

Some authors make the common mistake of mixing some of the Results in this section. You need to avoid such a mistake.

To be sure of the adequacy of this section, ask a colleague to read the Materials and Methods section. It is usual that your colleague will find an error or missing information in that section, simply because you were too close to your work. It is usual for someone who successfully does an experiment to find some difficulty explaining it with the same success.

\section{How to write the "Results"?}

The Results section is the core of the paper. It is the data. There are usually two ingredients of the Results section.

First, you should give some kind of overall description of the experiments, providing the "big picture", without, however, repeating the experiments details previously provided in Materials and Methods. Second, you should present the data. The results should be presented in the past tense. Of course it is not quite easy as there are several methods of presenting the data. It is essential to choose the most suitable method to present your data, and the best way depends on the type of data and the method of research.

Nevertheless, the manuscript should present the data in a representative manner, not in a repetitive manner.

In this section of your paper sacrifices will have to be made as you are always going to have more data than you can publish. Few journal editors or their readers want to see raw data. Additionally, you may have routine data especially in clinical research that have little to add to your research. They should not be included in the study.

Aaronson (1977) said: " The compulsion to include everything, leaving nothing out, does not prove that one has unlimited information; it proves that one lacks discrimination."

Handling of numbers is the central issue in the Results section. Descriptive presentation and tabular or graphic presentation are the main instruments available for use. And both should be used for good presentation of data. But they should be used to present the data in a meaningful way. For the author to do such a task successfully, he or she needs to determine the variables and determinations that affect the experiment. The variables that were tested have had either affected the experiment or not. Those that affected the experiment are better tabulated or graphed, while those that did not affect the experiment are described in the text.

The most important characteristic of the results is clarity. The results must be short and clear without verbiage. Although the Results section of a paper is the most important part, it is often the shortest, particularly if it is preceded by a well written Materials and Methods section and followed by a well-written Discussion.

The Results need to be clearly and simply stated because it is the Results that constitute the new knowledge that you are contributing to the world.

The earlier parts of the paper (Introduction, 
Materials and Methods) are designed to tell why and how you got the Results; the later part of the paper (Discussion) is designed to tell what they mean.

Obviously, therefore, the whole paper must stand or fall on the basis of the Results. Thus, the Results must be presented with crystal clarity.

\section{How to write the "Discussion"?}

Discussion is the hardest section to write. It is where the author analyzes the Results. The relationship among the data presented in the Results section need to be found. Based on these data, conclusions out of the research have to be made. The Results should be put in perspective, considering the previous published results. The theoretical and practical implications should be outlined and recommendations should be proposed.

The essential features of the good discussion are as follows:

1-The principles, relationships, and generalizations shown by the results are presented bearing in mind that in the discussion we discuss and not recapitulate the results.

2- The internal consistency of the Results is looked for. The different components of the experiment have a logical sequence, and need to reach logical consequences. However, the logic here is a scientific logic. Sometimes, we need to find out the logic that governs the relationship between the methods and the results. This is a scientific law or rule. It is sometimes known before the research is conducted. In such a case, the experiment only aims to test it. Some other times, it has to be found. When a certain method leads to a certain result, we have to find out why. If we could not clearly know the answer to that question, which is common in medicine, we do not cover it up. We state the question and leave it for further research.

3- Point out any unsettled points. Define the inconsistencies. State the lack of correlations. If you meet an exception, underline it. Sometimes the negatives are more valuable than the positives. The defects may be a lead to more interesting research. Consequently, do not cover up the gaps.
4- Show how your Results and interpretations agree or contrast with previously published work.

5-Discuss the theoretical and practical implications of your work.

6- State your conclusions as clearly as possible.

7-Summarize your evidence for each conclusion. Never assume anything without substantial objective evidence.

In simple terms, the primary purpose of the discussion is to show the relationships among observed facts. At the end of it, the discussion section should give a short summary or conclusion regarding the significance of the work. The reader of a paper usually ends asking " So What?" The discussion should give a satisfactory answer to that question. If the reader still asks that question, or still has it in mind, then the discussion has failed to reach its purpose. Good writing, like good music, has to end in a climax. More importantly, the climax must come at the right time.

\section{Defining the scientific truth:}

In showing the relationships among observed facts, you do not need to reach cosmic conclusions. Seldom, will you be able to illuminate the whole truth; more often, the best you can do is shine a spotlight on one area of the truth. Your one area of the truth can be illuminated by your data; if you attempt to use your data to draw a bigger picture than that shown by these data, it may lead the readers to confusion to the degree of doubting your data-supported conclusions.

Therefore, it is essential to exhibit your bit of truth. And do it simply. The simplest statements evoke the most wisdom, while complex language and complicated technical words are usually used to convey shallow thought.

\section{How to write the "References"?}

There are far more mistakes in the Reference section of a paper than anywhere else. At the same time the mistakes in the Reference section are generally solid, unforgivable, and always preventable. One of the mistakes is to cite references in the text and miss them in the References section. The reverse mistake is as common; to cite a reference without referring to it in the text. These mistakes occur as a 
result of addition and omission of references which is expected during drafting the paper. To avoid those two mistakes, it is essential to revise the reference section, which is also called the bibliography and the references in the text, and match the references in both.

\section{Reference styles:}

Although there are an almost infinite variety of reference styles, most journals cite references in one of three general ways that may be referred to as "name and year", "alphabetnumber", and "citation order".

\section{Name and year system:}

The name and year system has been very popular for many years and is used in many journals and books. Its big advantage is convenience to the author, as the references can be deleted and added easily. The disadvantages relate to the readers and publishers. The disadvantage to the readers occurs when a large number of references must be cited within one sentence or paragraph. Even two or three references cited together can be distracting to the reader. To the publisher, the disadvantage is increased cost.

Because some papers are written by a great number of authors, most journals that use name and year have an "et al." rule. Typically, it works as follows. Names are always used in citing papers with either one or two authors, e.g. "Smith (1998)," Smith and Jones (1998)." If the paper has three or more authors, it is cited as "Smith et al. (1998)."

In the references section some journals prefer that all authors be listed (no matter how many); other journals cite only the first three authors and follow with "et al."

\section{Examples are:}

Day R.A.1998. How to write and publish a scientific paper. 5th ed. Phoenix: Oryx Press.

Huth E.J. 1986. Guidelines on authorship of medical papers. Ann. Intern. Med. 104:269274.

\section{Alphabet-number system:}

This system, citation by number from an alphabetized list of references, is a modification of the name and year system. Citation by numbers keeps printing expenses within bound; the alphabetized list, particularly if it is a long list, is relatively easy for authors to prepare and readers (especially librarians) to use. Some authors who have habitually used the name and year system tend to dislike the alphabetnumber system, claiming that citation of numbers cheats the reader. The reader should be told, so the argument goes, the name of the person associated with the cited phenomenon; sometimes, the reader should also be told the date, on the grounds that an 1899 reference might be viewed differently than a 1999 reference.

\section{Examples are:}

1- Day R.A.1998. How to write and publish a scientific paper. 5th ed. Phoenix: Oryx Press.

2- Huth E.J. 1986. Guidelines on authorship of medical papers. Ann. Intern. Med. 104:269-274.

\section{Citation order system:}

The citation order system is simply a system of citing the references (by number) in the order that they appear in the paper. This system avoids the substantial printing expenses of the name and year system, and readers often like it because they can quickly refer to the references if they so desire in one-two-three order as they come to them in the text.

For long papers, the order system is problematic to the author because of the substantial renumbering associated chore that results from addition or deletion of references. However, the citation order system is now recommended by many journals. Preferably, the author has to consult the instructions for authors section, and have a close look at the journal before typewriting the final manuscript.

\section{Examples are:}

1- Huth EJ. Guidelines on authorship of medical papers. Ann Intern Med 1986; 104:269-74.

2- Day RA. How to write and publish a scientific paper. 5th ed. Phoenix: Oryx Press, 1998.

In addition to its nonalphabetical arrangement of references, the citation order system is markedly different from the others in its advocacy of eliminating periods after abbreviations (of journal titles, for example), periods after authors' initials, and commas after 
authors' surnames.

\section{Getting started:}

A few people find writing a paper an easy task first time out. As paper writing is different than anything else, it is expected to be hard to perform. But it is not hard to learn. As every skill we learn in surgery, it needs guidance, interest, dedication and practice.

The beginning is always the most difficult step. In that regard the best advice is start with what you have in mind. You have observations that you need to tell your colleagues, and the scientific community. You have a message out of these observations. Just write them. Put them on paper. Then outline a scenario to convince your colleagues of the validity of your results. Decidedly, get up one day and start writing the paper. No matter how scrambled and ill organized it will be, still it will give you a draft to work on. Most authors find it better and easier to work on a draft than start from scratch.

The first draft should carry the main point of the paper, the message of the paper, or the bottom line. The draft may be composed by free writing. But a good draft follows an outline and has a concept map.

Many authors write the Results first, followed by the methods, the discussion, the references and lastly the introduction.

Obvious enthusiasm for the subject matter and a belief in its importance are necessary elements of a strong manuscript. Clear writing begins with focusing this enthusiasm, however. Focus implies a well-defined and clinically valid question, around which the manuscript is built. The introduction of the paper poses the question and asserts its importance, the Methods detail the strategy used to answer the question, the Results section provides the raw answers, and the Discussion places the answers into perspective.

Once the focus of the paper is identified, an outline can be set up for each section of the paper (Introduction, Methods, Results, Discussion) to provide the logical structure for the reader.

While an outline provides skeletal structure around which the text can be written, some authors perform better using free-flowing or "brainstorming" techniques. Using this method, many authors feel more at liberty to tell the "story" of the project in a smooth and hopefully captivating style.

Regardless of the technique used to jumpstart the writing process, the most important step is to begin putting words down so that the editing process can begin.

Most people explain things better when they are talking than when they are writing. Try writing the first draft the way you would talk. Do not worry about minor issues like repeating the same word. Put the draft aside for a week. Then read it again. You will be surprised how much easier it is to spot the parts that are difficult to understand.

\section{Finding an end:}

This ends that review article but not the answers to the question "how to write a paper?" Many other advices can be given about paper writing. I believe another review article can deal with some of them.

\section{References:}

1- Debehnke DJ, Kline JA, Shih RD: Research Fundamentals: Choosing an Appropriate Journal, Manuscript Preparation, and Interactions with Editors. Academic Emergency Medicine 2001; (8): 844-850.

2- Sharp D: Kipling's Guide to Writing a Scientific Paper. Croatian Medical Journal 2002; 43(3):262-267

3- Cunningham SJ: How to write a paper. $J$ Orthodontics 2004; (1): 47-51.

4- Day RA: How to write and publish a scientific paper. Phoenix: Oryx Press. 5th edn.1998 